\title{
Anti-HB-EGF Monoclonal Antibody KHK2866
}

National Cancer Institute

\section{Source}

National Cancer Institute. Anti-HB-EGFMonoclonal Antibody KHK2866. NCI Thesaurus. Code C95721.

A proprietary fucose-free monoclonal antibody directed against human heparin-binding EGF-like growth factor (HBEGF) with potential antineo plastic activity. Anti-HB-EGF Monoclonal Antibody KHK2866 binds to HBEGF, thereby blocking its binding to the EGF receptors. This prevents EGF receptor activation and the subsequent induction of cell growth signaling. HBEGF is mitogenic for fibroblasts and smooth muscle and may be involved in macrophage-mediated cellular proliferation. The fucose-free monoclonal antibodies enhance antigen dependent cellular cytotoxicity (ADCC), and increase binding affinity to the Fc Receptor to overcome genetic polymorphism. 